\title{
THE TOPOLOGY OF COMPLETE ONE-ENDED MINIMAL SURFACES AND HEEGAARD SURFACES IN $\mathbf{R}^{3}$
}

\author{
CHARLES FROHMAN AND WILLIAM H. MEEKS III
}

In this note we announce two fundamental theorems on the topological uniqueness of certain surfaces in $\mathbf{R}^{3}$. The first of these theorems, which will depend on the second theorem, shows that a properly embedded minimal surface in $\mathbf{R}^{3}$ with one end is unknotted. More precisely,

Theorem 1.1. Two properly embedded one-ended minimal surfaces in $\mathbf{R}^{3}$ of the same genus are ambiently isotopic ${ }^{1}$.

Theorem 1.1 was conjectured by Frohman [4] who proved it in the case that the surfaces are triply periodic. A result of Callahan, Hoffman, and Meeks (Corollary 2 in [1]) states that a doubly periodic minimal surface has one end and infinite genus, when it is not a plane. Their result and Theorem 1.1 have the following corollary.

Corollary 1.1. Any two properly embedded nonplanar minimal surfaces in $\mathbf{R}^{3}$ that are invariant under at least two linearly independent translations are ambiently isotopic.

Essential in understanding the uniqueness theorems described here is the concept of a Heegaard surface in a noncompact threemanifold, which generalizes the usual notion of a Heegaard surface $M$ in a closed three-manifold $N^{3}$. Recall that a compact embedded surface $M$ is called a Heegaard surface if it separates $N^{3}$ into two genus- $g$ handlebodies where $g$ is the genus of $M$.

Received by the editors November 7, 1989.

1980 Mathematics Subject Classification(1985 Revision). Primary53A10,57M99.

The first author was supported by the National Science Foundation grant DMS8701736, and the second author, by research grant DE-FG02-86ER250125 of the Applied Mathematical Science subprogram of the Office of Energy Research, U.S. Department of Energy, and National Science Foundation grant DMS-8900285.

${ }^{1}$ Two surfaces in $\mathbf{R}^{3}$ are ambiently isotopic if and only if there exists a oneparameter family of diffeomorphisms of $\mathbf{R}^{3}$ taking one surface to the other. 


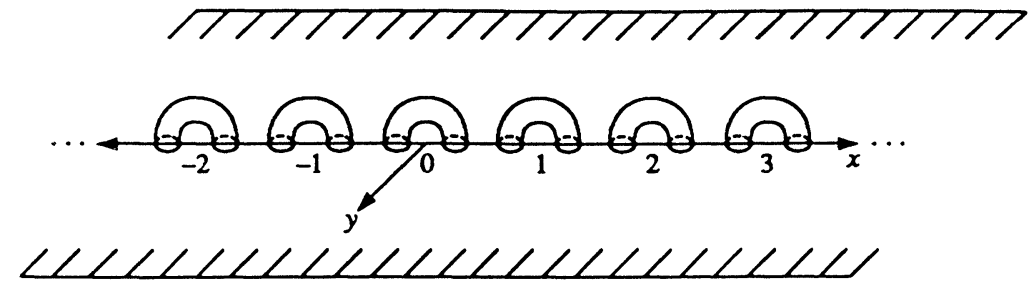

FIGURE 1

Noncompact three-manifolds such as $\mathbf{R}^{3}$ fail to have compact Heegaard surfaces. However, there is a natural notion of Heegaard surface for these manifolds where the surface is allowed to be noncompact. We say that a properly embedded surface $M$ is a Heegaard surface in $\mathbf{R}^{3}$ if the closures of each of the two components of $\mathbf{R}^{3}-M$ are handlebodies ${ }^{2}$.

We show that these handlebodies are each diffeomorphic to the three-manifold constructed as follows. Attach $g$ trivial onehandles to the closed lower half-space $H$ in $\mathbf{R}^{3}$ where $g$ is the genus of $M$, possibly infinite. When $g=\infty$, this attaching of handles on $H$ can be performed on neighborhoods of the integer points on the $x$-axis contained in $\partial H$ to obtain a one-periodic Heegaard surface in $\mathbf{R}^{3}$ (see Figure 1). Note that this implies that a Heegaard surface in $\mathbf{R}^{3}$ has one end.

Our second main theorem is

Theorem 1.2. Heegaard surfaces of the same genus in $\mathbf{R}^{3}$ are ambiently isotopic. Equivalently, given two diffeomorphic Heegaard surfaces in $\mathbf{R}^{3}$, there exists a diffeomorphism of $\mathbf{R}^{3}$ that takes one surface to the other surface.

Before discussing their proofs we wish to put Theorems 1.1 and 1.2 in historical perspective. First Waldhausen [14] proved the topological uniqueness of Heegaard surfaces in the unit threesphere $S^{3} \subset \mathbf{R}^{4}$. Later Lawson [9], using an argument of Frankel [2], proved that two closed minimal surfaces of genus $g$ in $S^{3}$ are Heegaard surfaces and hence isotopic by Waldhausen's theorem. Meeks [10] also proved some related topological uniqueness theorems for compact minimal surfaces with boundary in $\mathbf{R}^{3}$. More recently, Meeks and Yau [12] proved a topological uniqueness re-

\footnotetext{
${ }^{2} \mathrm{~A}$ three-manifold with boundary is a handlebody if it is homeomorphic to a closed regular neighborhood of a properly embedded one-dimensional $C W$ complex in $\mathbf{R}^{3}$.
} 
sult for properly embedded minimal surfaces that is closely related to Theorem 1.1. Their main theorem states that two proper diffeomorphic minimal surfaces in $\mathbf{R}^{3}$ of finite topology are ambiently isotopic.

We now give a brief outline of the proofs of Theorems 1.1 and 1.2. The main step in the proof of Theorem 1.2 is to show that for every compact subdomain $\Delta$ of a Heegaard surface $M \subset \mathbf{R}^{3}$, there exists a compact ball $B \subset \mathbf{R}^{3}$ with $\Delta \subset \operatorname{Int}(B)$ and $\partial B$ intersects $M$ transversely in a single simple closed curve. The proof of this step is nontrivial and is proved in part by generalizing a technique due to Haken in his study of Heegaard splittings of reducible three-manifolds [7]. The existence of $B$, together with Waldhausen's [14] uniqueness of Heegaard splittings of a ball with one boundary curve, implies there exists an exhaustion by balls $B_{1} \subset B_{2} \subset \ldots$ of $\mathbf{R}^{3}$ such that $\partial B_{i} \cap M$ is a simple closed curve, $B_{1} \cap M$ is a standard genus-one splitting of $B_{1}$, and $\overline{B_{i+1}-B_{i}} \cap M$ is a standard genus-one splitting of $\overline{B_{i+1}-B_{i}}$. This result implies $M$ is unique up to ambient isotopy.

Theorem 1.1 follows from Theorem 1.2 by proving that a properly embedded minimal surface $M \subset \mathbf{R}^{3}$ with one end is a Heegaard surface. Suppose $M$ is not a Heegaard surface. Then the closure $N$ of one of the complements of $M$ is not a handlebody. In this case we prove that there must exist a simple closed curve $\gamma \subset M$ that separates $M=\partial N$ and such that $\gamma$ is the boundary of a properly embedded noncompact stable minimal surface $\Sigma \subset N$ of finite total curvature and $\Sigma$ is incompressible in $N$. The proof of the existence of $\Sigma$ is delicate. It relies heavily on the curvature estimates of Schoen [13], the ability to minimize the area of a compact incompressible surface in its isotopy or homotopy class to acquire an embedded least-area surface [3,11], and the half-space theorem [8] that states that a properly immersed minimal surface contained in a half-space must be a plane. However, using the asymptotic behavior of a finite total curvature surface in $N$, it is easily shown that no such surface $\Sigma$ can be incompressible when $M=\partial N$ has one end. This contradiction completes the proof that $N$ must be a handlebody and therefore $M$ is a Heegaard surface. These results appear in [6].

We have also developed some theoretical results to deal with the topology and asymptotic behavior of properly embedded minimal surfaces in $\mathbf{R}^{3}$ with more than one end [5]. This work is based 
on an ordering theorem for the ends of such a surface. Loosely speaking our main theorem states that, after a rotation of $\mathbf{R}^{3}$, the ends of the surface can be ordered by their heights over the $\left(x_{1}, x_{2}\right)$-plane. In order to make precise the statement of the theorem, one needs the concept of a limit tangent plane for a properly embedded minimal surface. This definition, as well as the proof of existence and uniqueness of the limit tangent plane when the minimal surface has more than one end, is given in [1].

Theorem 1.3 (Ordering Theorem). Suppose $M$ is a properly embedded minimal surface in $\mathbf{R}^{3}$ with more than one end and whose limit tangent plane is the $\left(x_{1}, x_{2}\right)$-plane. Then the ends of $M$ are naturally ordered by their "height" over the $\left(x_{1}, x_{2}\right)$-plane.

We then go on to prove that the above ordering is a topological one:

Theorem 1.4. Suppose $M_{1}$ and $M_{2}$ satisfy the hypotheses of $M$ in Theorem 1.3 and $F: \mathbf{R}^{3} \rightarrow \mathbf{R}^{3}$ is a diffeomorphism such that $F\left(M_{1}\right)=M_{2}$. Then $F$ preserves or reverses the natural ordering of the ends of $M_{1}$ and $M_{2}$. In particular, if $M$ satisfies the hypotheses of Theorem 1.3 and $F: \mathbf{R}^{3} \rightarrow \mathbf{R}^{3}$ is a diffeomorphism such that $F(M)=M$, then $F$ preserves or reverses the ordering of the ends of $M$.

Conjecture 1.1. The ordering of ends of $M$ given in Theorem 1.3 is almost a well-ordering in the sense that it is equivalent to the ordering on a closed subset $\mathscr{S}$ of the interval $[0,1]$ with $\mathscr{S} \cap(0,1)$ discrete. In particular, $M$ can only have a countable number of ends.

The ordering theorem is proved by first showing that the ends of the surface $M$ can be separated by a collection, possibly infinite, of pairwise disjoint stable minimal surfaces $\Sigma_{i}$, each of which is asymptotic to an end of a plane or catenoid. Since the $\Sigma_{i}$ are disjoint and asymptotic to planes and ends of catenoids, eventually they are "parallel." We use this to define an ordering of the ends of $M$. The construction of the surfaces $\Sigma_{i}$ is based on earlier work in $[1,12]$.

We would like to thank Bob Edwards for his interest in Theorems 1.1 and 1.2, as well as useful conversations related to their proofs. 


\section{REFERENCES}

[1] M. Callahan, D. Hoffman, and W. H. Meeks III, The structure of singlyperiodic minimal surfaces, Invent. Math. 99 (1990), 455-481.

[2] T. Frankel, On the fundamental group of a compact minimal submanifold, Ann. of Math. 83 (1966), 68-73.

[3] M. Freedman, J. Hass, and P. Scott, Least area incompressible surfaces in 3-manifolds, Invent. Math. 71 (1983), 609-642.

[4] C. Frohman, The topological uniqueness of triply-periodic minimal surface in $\mathbf{R}^{3}$, J. Differential Geom. 31 (1990), 277-283.

[5] C. Frohman and W. H. Meeks III, The ordering theorem for the ends of properly embedded minimal surfaces, preprint.

[6] _ The topological uniqueness of complete one-ended minimal surfaces and Heegaard surfaces in $\mathbf{R}^{3}$, preprint.

[7] W. Haken, Some results on surfaces in 3-manifolds, Studies in Modern Topology, 1968.

[8] D. Hoffman and W. H. Meeks III, The strong halfspace theorem for minimal surfaces, Invent. Math. 5, 39-98.

[9] H. B. Lawson, The unknottedness of minimal embeddings, Invent. Math. 11 (1970), 183-187.

[10] W. H. Meeks III, The topological uniqueness of minimal surfaces in threedimensional Euclidean space, Topology 20 (1981), 389-410.

[11] W. H. Meeks III, L. Simon, and S. T. Yau, The existence of embedded minimal surfaces, exotic spheres and positive Ricci curvature, Ann. of Math. 116 (1982), 221-259.

[12] W. H. Meeks III and S. T. Yau, The topological uniqueness theorem of complete minimal surfaces of finite topological type, preprint.

[13] R. Schoen, Estimates for stable minimal surfaces in three dimensional manifolds, Ann. of Math. Stud., vol. 103, Princeton Univ. Press, Princeton, N.J., 1983, 111-126.

[14] F. Waldhausen, Heegaard zerlegungen von der drie-sphaere, Topology 7 (1968), 195-203.

Mathematics Department, SUNY at Buffalo, Buffalo, New York 14214

Mathematics Department, University of Massachusetts, Amherst, MASSACHUSETTS 01003 
